\title{
Theodor Kocher und die Anfänge der Radiologie in der Schweiz
}

von Constant Wieser

\section{ZuSAMMENFASSUNG}

Kocher hat schon im Januar 1896 die erste diagnostische Röntgenaufnahme veranlasst; der Physikprofessor Aimé Forster führte sie aus. Gemeinsam planten Forster und Kocher das Röntgeninstitut des Inselspitals, das am 1. Januar 1898 seinen Betrieb aufnahm.

Ausgangspunkt dieser Skizze ist eine kurze Notiz im Correspondenz-Blatt für Schweizer Ärzte, Vorläufer der Schweiz.medizinischen Wochenschrift, vom 15. Februar 1896. Dort wurde unter dem Titel «Die Röntgen'schen Experimente mit Kathodenstrahlen» erstmals über die Anwendung der X-Strahlen in der Medizin referiert. Die Entdeckung Röntgens war zwar auch in der Schweiz bereits früher durch die Tagespresse bekannt geworden, so durch einen eingehenden Artikel in der Neuen Zürcher Zeitung vom 10.Januar 1896.

In der erwähnten redaktionellen Zusammenfassung von verschiedenen Mitteilungen in ausländischen Zeitschriften wird beiläufig mitgeteilt :

«In Bern ermittelte Kocher auf diesem Weg den Sitz einer in der Hand steckenden Nadel.»

Interessant ist der Weg, auf dem diese Nachricht an die Schweizer Ärzte gelangte. Sie stammt aus dem Lancet vom 1. Februar 1896 und war über Berlin nach London gelangt.

Dieser Erstmeldung über die Anwendung der Röntgenstrahlen durch Kocher folgt, was seine Person betrifft, ein auffallendes Schweigen. Im Correspondenz-Blatt wird der Name Kochers in den nächsten Jahren im Zusammenhang mit dem Röntgenverfahren nicht mehr erwähnt. Auch gibt es weder bei Bonjour noch in der Bibliographie bei Tröhler direkte Hinweise für eine besondere Beschäftigung des späteren Nobelpreisträgers mit Problemen der Röntgendiagnostik oder -therapie. So stellt sich die Frage, ob dieser Erstmeldung eine Pioniertat Kochers zugrunde liegt, wie Verfasser in 
«Radiologie in der Schweiz» vielleicht etwas voreilig annimmt, oder war lediglich der internationale Bekanntheitsgrad des Operateurs der Grund für die Erwähnung im Lancet?

Und muss man vielleicht sogar das nachfolgende Schweigen dahin interpretieren, Kocher sei später auf Distanz zur neuen Methode gegangen? Hatte er doch unmittelbar vor der Entdeckung Röntgens seine «Beiträge zur Kenntnis einiger praktisch wichtiger Fracturformen» veröffentlicht. Darin kommt Kochers subtile klinische Untersuchungsmethode besonders schön zum Ausdruck.

Kehren wir zurück zur Meldung im Correspondenz-Blatt. Diese könnte vermuten lassen, Kocher hätte die Aufnahme selber hergestellt, was kaum der Fall sein kann. Wir wissen, dass seit dem Bekanntwerden der Röntgenphotographie sich überall in unserem Land Physiker rasch der Methode angenommen haben. So auch in Bern. Hier hielt Aimé Forster (1843-1926), Professor für Physik, am 27. Januar 1896 - einen Monat nach Bekanntwerden der «Vorläufigen Mitteilung» Röntgens - anhand eigener Beobachtungen vor dem Medizinisch-Pharmazeutischen Bezirksverein darüber einen Vortrag. Die entsprechende Notiz im Correspondenz-Blatt gibt keine weitere Auskunft über die Ausführungen von Prof. Forster noch über die, wie es dort heisst, gelungenen Versuche.

Forster hat aber im Herbst 1896 seine ersten «Radiodiagnostischen Erfahrungen» in einer 15seitigen Broschüre - wie es in Glasers Bibliographie bescheiden heisst - veröffentlicht. Die Einsichtnahme in das einzige, uns bekannte Exemplar war für unsere Fragestellung sehr aufschlussreich. Ich danke dem Berner Physikalischen Institut und Herrn Prof. Boschung, mir diese ermöglicht zu haben. Denn dem kurzen Text mit Diagnose und Herkunft der untersuchten Patienten folgen 32 Tafeln mit Kopien seiner Originalröntgenplatten. Nach dem Vorwort hat Prof. Forster diese selber angefertigt und eingeklebt. Leider sind keine Aufnahmedaten vermerkt. Doch können wir annehmen, die Abbildungen seien chronologisch geordnet.

Das dritte Bild im Kapitel «Missbildungen und Fremdkörper» dürfte die im Lancet erwähnte Aufnahme der Nadel in der Hand einer jungen Dame sein, die Kocher dann chirurgisch entfernt hat.

Tröhler erwähnt in seiner Kocherbiographie Kochers Zusammenarbeit mit dem Physiker Forster am Beispiel der Lokalisationsmethode von metallischen Fremdkörpern mittels eines Riesengalvanometers. Die Entstehungsgeschichte dieser Methode schildert Forster selber ausführlich im Nachwort der erwähnten Schrift. Auch verweist Tröhler auf die ballistischen Berech- 
nungen Forsters für Kochers Studien über die Pathophysiologie der Geschosswirkung. Weder bei Bonjour noch bei Tröhler ist aber expressis verbis die Zusammenarbeit bei Röntgenuntersuchungen erwähnt. Bei den Schiessversuchen 1896 wurden auch Röntgenstrahlen angewandt, wie Abbildung 19 der Sammlung mit der Wirkung einer Schussverletzung durch den Oberschenkel einer Leiche zeigt.

Dass Kocher sehr früh die diagnostischen Möglichkeiten der Röntgenstrahlen auch bei klinischen Fragestellungen ausschöpfte, so bei der Knochen- und Gelenktuberkulose, die wenige Jahre später zu Kontroversen mit seinen beiden Schülern Oscar Bernhard (1861-1939) und Auguste Rollier (1874-1954) führen sollten, beweist die Aufnahme einer Spina ventosa.

Im gedruckten Jahresbericht der Insel-Korporation von 1896 wird das Röntgenverfahren noch nicht erwähnt, wohl aber im nachfolgenden Jahr. Am 29. Mai 1897 hat die Spitaldirektion an den Verwaltungsrat den Antrag zur Errichtung eines eigenen Röntgeninstitutes am Inselspital gestellt. Der von Hans Schmocker aufgearbeiteten Korrespondenz im Berner Staatsarchiv, die zu dieser Eingabe geführt hatte, entnehmen wir,

«dass Prof. Forster sich sehr früh aus wissenschaftlichem Interesse mit der von Prof. Röntgen Ende 1895 entdeckten Skiographie befasste. Ab März 1896 habe er dann sein Physikalisches Institut dem Publikum bereitwilligst und unentgeltlich zur Verfügung gestellt. Dies sei jetzt aber nicht mehr möglich.»

Bis zum 15. Februar 1897 hatte Forster 506 Röntgenaufnahmen angefertigt. Von den 85 Patienten, die aus dem Inselspital ins Physikalische Institut gebracht wurden, kamen 57 aus der Klinik von Prof. Kocher. Bei diesen wurden 121 Aufnahmen angefertigt. So überrascht es uns nicht, dass sich Kocher zusammen mit seinem Kollegen Forster um die Einzelheiten der Einrichtung und Organisation eines eigenen Institutes am Inselspital bemühte. Dieses nahm am 1.Januar 1898 seinen regulären Betrieb auf. Von den 1898 dort angefertigten 580 Aufnahmen waren zwei Drittel für die Kochersche Klinik bestimmt.

Bei diesen Akten liegt auch eine Meldung über Röntgentaxen in Chur, wo der Kleine Rat bereits am 30. Januar 1897 die Einrichtung eines Röntgenzimmers im Physikgebäude der Kantonsschule beschlossen hatte.

Auch finden wir hier ein sympathisches Schreiben vom Oktober 1899 an Dr.Schenkel, Leiter des Röntgens am Inselspital, worin Kocher sich bereit erklärt, bei Regierungsrat Gobat auf die Notwendigkeit einer Vergrösserung und Bessereinrichtung der Röntgenräume hinzuweisen. Das entsprechende Schreiben ist ebenfalls vorhanden. 
Nach diesen Hinweisen dürfen wir die eingangs gestellte Frage wohl dahin beantworten, dass Theodor Kocher rasch die Bedeutung der Röntgenstrahlen für die Medizin erkannt und sie bei seinen Patienten auch angewandt hat. Es gehörte zur Bescheidung seines Genies, die Anwendung der Hilfswissenschaften dann kompetenten Fachvertretern zu überlassen.

Zum Schluss sei noch ein Hinweis auf den indirekten Beitrag von Theodor Kocher zur späteren Entwicklung der Radiologie in der Schweiz erlaubt. Eine Reihe seiner Schüler, so sein Nachfolger Fritz de Quervain (1868-1940), gehörten 1913 zu den Gründern der Schweizerischen Röntgengesellschaft. Ein Hauptinitiant und der führende Kopf in dieser Aufbauphase war Dr. Hermann Hopf (1874-1930). Im Nachruf in der Schweiz.med. Wochenschrift 1930 lesen wir :

«Sein von ihm hochverehrter Lehrer und einstiger Chef Theodor Kocher hatte, den Wert und die Entwicklungsmöglichkeiten der Entdeckung Röntgens für die Chirurgie und medizinische Diagnostik erkennend, mit sicherem Blick in Dr. Hopf die zur Ausübung und weiteren Entwicklung dieses neuen Hilfsmittels geradezu prädestinierten Fähigkeiten erkannt und ihn ermuntert, sich dem Röntgenfach zu widmen. Dr. Hopf folgte Kochers Rat. Nach kurzer Ausbildung in Aschaffenburg zog er 1910 nach Bern, wo er das erste private Röntgeninstitut eröffnete.»

Die sich hier aufdrängende Frage, warum Hopf nicht ins Inselspital berufen und von Kocher nicht zum Aufbau eines Universitäts-Röntgeninstitutes ermuntert wurde, gehört nicht mehr zum Thema dieser Skizze, wäre aber einer eigenen Untersuchung wert.

Für den Quellennachweis konsultiere man: C. Wieser, H. Etter, J. Wellauer, Radiologie in der Schweiz, Bern (Huber) 1989. Vgl. die Buchbesprechung in Gesnerus 49 (1992), Part 1, S. $107 \mathrm{f}$. 


\title{
Summary
}

\section{Theodor Kocher and the beginnings of radiology in Switzerland}

Already in January 1896, Kocher ordered his first clinical radiography. It was executed by Aimé Forster, professor of physics at the University ob Berne. Together, Forster and Kocher elaborated the plans for the Röntgen Institute of the Inselspital, which opened on January $1^{\text {st }}$, 1898.

\section{Résumé}

\section{Theodor Kocher et les débuts de la radiologie en Suisse}

Déjà en janvier 1896, Kocher demanda une première radiographie clinique à son collègue Aimé Forster, professeur de physique à l'Université de Berne. En collaboration édroite, Forster et Kocher dressèrent les plans pour l'Institut Roentgen à l'Hôpital de l'Ile, qui entra en activité le l $^{\text {er }}$ janvier 1898 .

\author{
Dr.med.Constant Wieser \\ Campodelsweg 23 \\ 7000 Chur
}

